Cahiers $d u$ MONDE RUSSE

\section{Cahiers du monde russe}

Russie - Empire russe - Union soviétique et États indépendants

$44 / 4 \mid 2003$

Varia

\title{
Paul Aron, Frédérique Matonti, Gisèle Sapiro, eds., «Le réalisme socialiste en France »
}

\section{Catherine Depretto}

\section{OpenEdition}

\section{Journals}

Édition électronique

URL : https://journals.openedition.org/monderusse/4145

DOI : 10.4000/monderusse.4145

ISSN : $1777-5388$

Éditeur

Éditions de l'EHESS

Édition imprimée

Date de publication : 1 octobre 2003

ISBN : 2-7132-1833-0

ISSN : $1252-6576$

Référence électronique

Catherine Depretto, «Paul Aron, Frédérique Matonti, Gisèle Sapiro, eds., "Le réalisme socialiste en France » », Cahiers du monde russe [En ligne], 44/4 | 2003, mis en ligne le 19 juin 2009, consulté le 03 septembre 2022. URL : http://journals.openedition.org/monderusse/4145 ; DOI : https://doi.org/ $10.4000 /$ monderusse. 4145

Ce document a été généré automatiquement le 3 septembre 2022

Tous droits réservés 


\title{
Paul Aron, Frédérique Matonti, Gisèle Sapiro, eds., " Le réalisme socialiste en France »
}

\author{
Catherine Depretto
}

\section{RÉFÉRENCE}

Paul ARON, Frédérique MATONTI, Gisèle SAPIRO, eds., « Le réalisme socialiste en

France ». Sociétés \& Représentations, 15, 2002, 424 p.

1 Ce volume est le résultat d'une collaboration engagée entre le Centre de sociologie européenne (CNRS-EHESS, Paris) et le Centre d'histoire de la littérature belge en langue française (Université libre de Bruxelles). Réunissant historiens, sociologues, politistes, historiens de la littérature et des arts plastiques des deux pays, il a été préparé par deux rencontres en 2000 et 2001 et coordonné plus spécialement par Paul Aron, spécialiste belge de littérature prolétarienne et Gisèle Sapiro, connue pour ses études sur les milieux littéraires français, inspirées des travaux de Pierre Bourdieu ${ }^{1}$.

2 La question du réalisme socialiste fait certainement partie des sujets qui, depuis 1991 et la fin de l'URSS, ont commencé à pouvoir être abordés de façon plus sereine et surtout avec une meilleure base documentaire. À la suite des travaux pionniers de Katerina Clark ou de Régine Robin, nombreux sont les ouvrages de référence parus ces dix dernières années (Antoine Baudin, Evgenij Dobrenko, Hans Günther, Leonid Heller, Vladimir Papernyj...) et dont Michel Aucouturier présente dans ce volume une éclairante synthèse ${ }^{2}$. Mais la question a aussi largement bénéficié du développement récent de certaines problématiques, extérieures au strict domaine des études littéraires, comme celles qui touchent en France à l'histoire comparative des intellectuels, aux transferts culturels, aux rapports entre art et politique, à l'histoire du communisme. Aussi la question du réalisme socialiste, loin de se limiter à l'URSS ou aux démocraties populaires, peut-elle se révéler un sujet fécond pour d'autres pays dont la France, non 
que la question n'ait été abordée auparavant, mais elle peut aujourd'hui être l'objet d'une approche renouvelée et interdisciplinaire, grâce précisément au développement de ces champs d'étude. Et c'est bien dans cette double perspective qu'il faut lire le travail de questionnement auxquels les auteurs du volume ont soumis, chacun selon ses compétences propres, la notion de réalisme socialiste en France, refusant de considérer comme définitivement élucidé ce phénomène somme toute marginal de la culture française qu'aux dires de certains « seul l'égarement militant des communistes avait pu prendre au sérieux $»^{3}$.

Derrière les différentes contributions, on sent une même volonté de reprendre à la base un certain nombre de faits, de les réexaminer sans a priori, de faire voir la réalité concrète, les implications de certains choix, sans pour autant négliger l'apport historiographique de travaux antérieurs ${ }^{4}$; l'entreprise offre surtout une alternative à l'opposition qui continue trop souvent à renvoyer dos à dos les partisans d'une lecture strictement politique et idéologique du réalisme socialiste et ceux qui insistent pour dégager un canon esthétique.

Le réalisme socialiste est ici principalement questionné en termes d'histoire sociale, prenant en compte sa dimension de phénomène d'importation et l'état de la culture d'accueil au moment de sa réception, et comme renvoyant ensuite à des positionnements dans le champ littéraire et politique, comme révélateur de stratégies individuelles ou collectives, pouvant obéir à différentes logiques, d'où un axe à la fois historique (des années 1930 à la guerre froide) et thématique.

5 L'approche historique permet de bien suivre l'évolution de la notion, depuis son introduction timide dans les années 1930 jusqu'à son abandon dans les années 1960 et de repérer le rôle central joué par la période de la guerre, à la fois dans la redéfinition même du réalisme socialiste et surtout dans le changement des stratégies du parti communiste français à l'égard des intellectuels. Par ailleurs, à l'intérieur de cette structuration chronologique, des études de cas ou l'examen de parcours individuels permettent de montrer la diversité des contenus, renvoyant au réalisme socialiste à différentes périodes. Il pourrait être fastidieux de rendre compte séparément de toutes les contributions, d'autant plus qu'il y a parfois entre elles des recoupements inévitables, même si les perspectives ne sont pas toujours identiques. Il m'a semblé plus intéressant de repérer quelques-unes des lignes de force qui se dégagent de l'ensemble et surtout d'insister sur ce qui, d'un point de vue méthodologique et conceptuel, peut avoir de l'importance pour les slavistes et pour l'étude du réalisme socialiste soviétique.

On retire tout d'abord l'impression que le réalisme socialiste, introduit dans la presse communiste fin 1932, mais surtout après le Premier congrès des écrivains soviétiques en 1934 (voir l'article de Philippe Baudorre5), n'a pas véritablement donné lieu en France à un débat d'une quelconque ampleur; le corpus des textes français sur la question auquel il est fait référence reste en définitive assez mince et de peu d'intérêt, ce qui constitue une différence notable avec d'autres pays, par exemple la Tchécoslovaquie de l'entredeux-guerres et la réflexion d'un artiste comme Karel Teige. Compris comme la nécessité de produire un art de parti, le réalisme socialiste a été l'objet de fortes résistances en France, aussi bien de la part d'écrivains prolétariens, populistes, révolutionnaires, que de communistes ou compagnons de route qui, comme Jean-Richard Bloch, n'ont jamais caché leur opposition à ce mot d'ordre et défendu l'expérimentation en art (voir l'article de Michel Trebitsch). On remarque une même réticence chez les peintres, en particulier chez Fernand Léger, qui s'oppose à Aragon et se refuse à renoncer aux avancées les plus 
décisives de l'art pictural d'avant-garde au nom d'un problématique " retour au sujet » imposé par l'adhésion au réalisme socialiste. En fait, pour les artistes français engagés dans le contexte bien spécifique du Front populaire, le ralliement ne se fait pas autour du réalisme socialiste, mais autour d'un unanimisme de défense de la culture, comme le montre Nicole Racine dans son article sur la « querelle du réalisme (1935-1936) ». Ainsi certains traits spécifiques à la France expliqueraient peut-être en partie cette difficile acclimatation du réalisme socialiste dans les années 1930 : le contexte particulier du Front populaire, joint à l'absence de politique clairement définie en direction des artistes de la part du parti communiste français.

7 C'est là l'importance de l'approche historique, qui montre comment la situation évolue avec la guerre et surtout l'après-guerre où, avec la guerre froide, l'alignement sur le jdanovisme culturel soviétique se révèle beaucoup plus contraignant pour les artistes communistes (articles de Gisèle Sapiro, "Formes et structures de l'engagement des écrivains communistes en France : de la "drôle de guerre" à la Guerre froide » et de Lucie Fougeron, "Un exemple de mise en images: le "réalisme socialiste" dans les arts plastiques en France, 1947-1954»). Cela dit, par-delà les évolutions historiques, chez des écrivains comme Paul Nizan (article de Sophie Cœuré sur ses récits d'URSS, avec un texte inédit sur Bakou) ou, dans un tout autre ordre d'idées, Pierre Courtade ${ }^{6}$, qui semblent avoir adopté le réalisme socialiste, il y a peut-être place pour des lectures non univoques. Certes, la France produit des textes qui rejoignent certains modèles soviétiques, comme les récits de vie (article de Bernard Pudal), ou, dans l'après-guerre, des romans qu'on peut, malgré leur diversité, qualifier de « réalistes socialistes » et dont Reynald Lahanque dresse la typologie ${ }^{7}$, mais dans l'un et l'autre cas il est bien difficile de mettre en évidence des traits qui renverraient à une écriture spécifiquement « réaliste socialiste »: on a, d'une part, affaire à un phénomène constitutif du communisme, l'obsession biographique, et, de l'autre, on retombe sur le modèle du roman à thèse, certes empreint d'une vision communiste stalinienne du monde (la guerre froide). Enfin, ce qu'on savait déjà, mais qui se trouve réaffirmé ici, c'est le rôle essentiel joué par Louis Aragon dans l'introduction du réalisme socialiste en France, au point qu'on peut se demander si la question a un sens hors de sa personne ${ }^{8}$. C'est Aragon d'abord qui lui donne un tour national, en l'ancrant dans la tradition du réalisme française et le définit de « façon tellement large qu'il devient difficile de comprendre ce qui le différencie d'un réalisme non socialiste $»^{9}$. Il place ensuite son œuvre et son parcours sous sa bannière, avec la série des œuvres sur le monde réel, mais surtout continue à s'en réclamer même après le $\mathrm{xx}^{\mathrm{e}}$ congrès du PCUS. Plutôt que d'oblitérer cette revendication insistante du réalisme socialiste, Philippe Olivera essaye d'en rendre compte en termes de stratégie personnelle, de construction biographique, mais on ne peut dire que toutes les interrogations soient vraiment levées et peut-être doit-on admettre qu'il restera là une zone d'ombre. Comme le rappelle en substance Reynald Lahanque, l'initiative d'Aragon en direction du réalisme socialiste ne " correspond ni à une volonté clairement affichée de la part des Soviétiques d'exporter le mot d'ordre inscrit dans les statuts de l'Union des écrivains, ni à une politique décidée par la direction du PCF $»^{10}$.

De l'ensemble de ces contributions ressort donc surtout, comme l'écrivent Paul Aron et Gisèle Sapiro, le caractère labile de la notion, qui évolue dans le temps mais surtout a du mal à trouver sa spécificité. C'est souvent ce qui se passe lorsqu'on regarde un phénomène dans ses manifestations concrètes, par en bas, mais cela renvoie aussi à une difficulté inhérente à la notion même: si on sait souvent ce que le réalisme socialiste n'est pas, on a en général plus de mal à dire ce qu'il est ; n'est-ce pas ce que déclarait 
Aragon lui-même, parlant d'un « désir d'unir pêle-mêle presque n'importe qui » et d'une formule permettant "d'annexer presque n'importe quoi » ${ }^{11}$ ? La question du réalisme socialiste français est donc loin d'être tranchée au terme de ces différentes études; comme l'indiquait clairement la présentation, il s'agissait plus pour les auteurs d'ouvrir un champ et d'explorer des pistes que d'apporter des réponses. De ce point de vue, on ne peut que relever la fécondité d'une approche interdisciplinaire et tout le profit qui résulte de la mise en commun de compétences complémentaires pour cerner un phénomène hétéronome par définition et qui a à voir avec plusieurs domaines, principalement l'art et la politique. Même s'il continue d'apparaître comme un espace second de la culture française, le réalisme socialiste français mérite sans doute qu'on s'y intéresse, ne serait-ce que parce qu'il touche des écrivains importants comme Aragon ou Nizan et éclaire certaines prises de position comme celles de Sartre ou de Roland Barthes dans les années 1950 (article de Benoît Denis, "Les écrivains engagés et le réalisme socialiste, 1944-1953»). Mais on pourrait aller plus loin et s'interroger davantage sur ce caractère second. Certes, il tient à la position marginale de ce qui relève de la sphère communiste dans le contexte culturel français, en particulier avant-guerre, mais le peu d'intérêt que suscite la question du réalisme socialiste chez les Français, y compris chez les intellectuels communistes, peut aussi nous orienter vers d'autres pistes. Si les Français ne passent pas trop de temps à s'interroger sur la question, c'est qu'ils ne sont pas dupes des mots et qu'ils ont compris d'emblée qu'on a affaire à un slogan politique, réclamant un art de parti, ce qu'ils ne souhaitent pas vraiment et qui ne correspond pas aux traditions culturelles françaises : l'engagement personnel ne signifie pas forcément art de parti. Gisèle Sapiro montre bien comment, dans l'après-guerre, on assiste sans doute à des tentatives de la part du PCF pour remplacer ce mode d'engagement propre aux intellectuels français (depuis l'affaire Dreyfus) par un modèle plus semblable à ce qui s'est mis en place en URSS et qui implique aussi la mobilisation des productions artistiques elles-mêmes, leur mise au service de la "tendance». Mais, même à ce moment, est maintenue la nécessité d'une critique littéraire de professionnels et les tentatives en direction des intellectuels non communistes se font toujours sur une base large; on parle plus de "littérature progressiste " que de "réalisme socialiste » ${ }^{12}$. Par conséquent, cela nous incline à penser que la "fortune » du réalisme socialiste est intrinsèquement liée à un fonctionnement institutionnel spécifique, celui d'une subordination totale de l'art au pouvoir par le truchement d'unions professionnelles, telles qu'elles sont mises en place en URSS dans le cadre d'un régime de nature totalitaire (les tentatives du PCF pour reproduire ce modèle dans l'après-guerre restent bien en deçà).

9 La difficulté à mettre en évidence une esthétique du roman réaliste socialiste français confirme la nature politique de l'expression et renvoie à une même difficulté inhérente au réalisme socialiste soviétique pour lequel, malgré la durée du phénomène et l'abondance des œuvres portant ce label, on a aussi bien du mal à définir un canon. Autre similitude entre les deux pays : il semble bien qu'en France les débats sur les questions d'art prolétarien, révolutionnaire, populiste, cessent à partir du moment où l'on commence à parler de réalisme socialiste et que certains auteurs, plutôt engagés du côté des expériences prolétariennes, esquissent un rapprochement avec le nouveau slogan (voir l'article de Jean-Charles Ambroise sur Tristan Rémy). Ne peut-on voir ici la confirmation même indirecte du caractère de "contre-révolution culturelle " qu'a signifiée en URSS l'adoption du réalisme socialiste ${ }^{13}$ qui à court terme libérait les compagnons de route du diktat des écrivains prolétariens, mais signifiait à plus longue 
échéance l'arrêt des expériences radicales en matière d'art pour le peuple? Enfin, dans les résistances rencontrées par le réalisme socialiste en France et l'absence de débat sur le sujet, dans l'indifférence manifestée par les artistes français à l'égard des œuvres soviétiques, ne peut-on voir la preuve que la culture et la littérature françaises restent dominantes? Les Français n'envisageraient pas un seul instant de pouvoir apprendre quoi que ce soit des Soviétiques sur un plan culturel, même s'ils en acceptent la prééminence politique par adhésion au communisme ${ }^{14}$.

10 La signification de ces " échanges inégaux » est d'ailleurs parfaitement bien montrée par Ioana Popa dans l'article qu'elle consacre aux traductions littéraires ${ }^{15}:$ l'intensité des flux de traduction des œuvres réalistes socialistes dépend de la position qu'occupent les littératures et les pays «source» et «cible» à la fois dans l'espace littéraire international et dans le système des relations politiques internationales. Ainsi, doublement satellisées, les démocraties populaires ne voient guère leurs écrivains réalistes socialistes profiter d'une quelconque politique de traduction; en revanche, elles accueillent généreusement les traductions d'œuvres soviétiques et offrent même à nos romanciers réalistes socialistes des possibilités inespérées de diffusion. Pour finir, signalons trois contributions qui examinent des épisodes liés à l'histoire du réalisme socialiste hors de France : l'exportation en Roumanie du modèle soviétique de contrôle des arts (Lucia Dragomir), les particularités du jdanovisme en République moldave (Petru Negura), la façon dont la question du réalisme socialiste s'est posée auprès des plasticiens belges dans l'après-guerre (Virginie Devillez).

11 En conclusion, cet ouvrage est une tentative réussie pour renouveler l'étude du phénomène qu'est le "réalisme socialiste ", grâce à une approche pluridisciplinaire et comparative ${ }^{16}$. Il montre tout le bénéfice qu'on peut tirer d'un traitement politique et sociologique de certains faits culturels et peut, de ce point de vue, offrir aux spécialistes de la période soviétique des perspectives de recherche. Malgré la très bonne présentation de Paul Aron et Gisèle Sapiro, il manque peut-être toutefois un article de synthèse, qui permettrait d'avoir une vision plus nette des principaux acquis de ce travail, mais, sans doute, était-il encore un peu trop tôt pour tirer des conclusions.

\section{NOTES}

1. Auteur en particulier d'un ouvrage important sur le champ littéraire français, La guerre des écrivains 1940-1953, Paris, Fayard, 1999, où elle montre entre autres que les clivages entre écrivains collaborationnistes et résistants ne sont pas le fruit du hasard, mais correspondent aux positionnements des uns et des autres dans le champ littéraire avant guerre.

2. «Du nouveau sur le "réalisme socialiste" ?", p. 363-368.

3. Paul Aron et Gisèle Sapiro, présentation, p. 5.

4. Comme celui bien connu de Jean-Pierre Morel, Le roman insupportable. L'Internationale littéraire et la France (1920-1932), Paris, Gallimard, 1985.

5. Philippe Baudorre, «Le réalisme socialiste français des années trente : un faux départ», p. 15-38. 
6. Voir respectivement Paul Aron, «Être ou ne pas être réaliste socialiste : l'exemple d'Elseneur de Pierre Courtade ", p. 217-228 et Frédérique Matonti, " "Il faut observer la règle du jeu". Réalisme socialiste et contrebande littéraire : La Place rouge de Pierre Courtade ", p. 293-306.

7. Enseignant la littérature comparée, il a soutenu en décembre 2002 une thèse de doctorat à l'Université de Nancy II, intitulée « Le réalisme socialiste en France 1934-1954 ».

8. Sa figure est ainsi présente dans un bon nombre de contributions, voir en particulier celles de Philippe Baudorre, Nicole Racine, Daniel Virieux, Gisèle Sapiro, Philippe Olivera.

9. Ph. Baudorre, art. cit., p. 38.

10. «Les romans du réalisme socialiste français », p. 177.

11. L. Aragon, Euvre poétique, t. 6, 1934-1935, Paris, Livre club Diderot, 1975, p. 193.

12. G. Sapiro, art. cit., p. 172.

13. Rappelons que la formule doit selon toute vraisemblance être attribuée à Stalin.

14. Voir ce qu'écrit Lucie Fougeron, art. cit., p. 203 : «On remarque par ailleurs la proportion relativement faible de toiles et de sculptures inspirées d'événements ou de personnages situés en URSS. Et si la presse communiste publie de nombreux articles louant le réalisme socialiste - et plus généralement la culture - soviétique, faisant écho à la glorification systématique de la "patrie du socialisme", il semble cependant que les peintres du "nouveau réalisme français" aient été assez indifférents à la production picturale soviétique, privilégiant, à l'exemple de Louis Aragon notamment, la caractère national inspiré de la tradition de l'art figuratif français. »

15. Sur la question, on consultera aussi : "Traduction : les échanges littéraires internationaux ", Actes de la recherche en sciences sociales, 144, septembre 2002.

16. On signalera parmi les coquilles l'attribution du prénom Constantin à Radek et Fréville, p. 17, 22, 121 ; en outre, et sauf erreur de notre part, ni André Gide ni Paul Nizan n'étaient présents au Premier congrès des écrivains soviétiques, p. 21, p. 79 note 10. 